\title{
PLACENTAL STEREOLOGY: SPANNING THE LEVELS FROM MOLECULE TO WHOLE ORGAN
}

\author{
TERRY M MAYHEW \\ School of Biomedical Science, E Floor, Queen's Medical Centre, University of Nottingham, Nottingham, NG7 \\ 2UH, UK \\ E-mail: terry.mayhew@nottingham.ac.uk \\ (Accepted July 29, 2009)
}

\begin{abstract}
Stereology can provide hard (functionally-relevant) quantitative information at different levels of 3D structural organization. My researches have applied established methods to study organ function and developed novel methods to study subcellular localization of marker probes. We have studied whole-organ function (passive diffusion) in the human placenta and quantified various processes at tissue and subcellular levels and in normal and complicated pregnancies. Fetoplacental angiogenesis and villous growth and maturation involve phased changes particularly around mid-gestation. Growth is associated with increased numbers of cells or nuclei and counts have shown that villous trophoblast continuously renews itself via cytotrophoblast (CT) proliferation and recruitment and syncytiotrophoblast (ST) differentiation and extrusion. Integration of these processes results in changes in total oxygen $\left(\mathrm{O}_{2}\right)$ diffusive conductance which match the growing fetal mass. Similar processes occur during development of the mouse placenta and at least some are compromised in human pregnancies. For example: [a] in pure pre-eclampsia (PE), villous and fetoplacental vascular volumes and surfaces are similar to those seen in uncomplicated pregnancies but reduced in pure intrauterine growth restriction (IUGR) and in PE+IUGR; [b] trophoblast extrusion is accelerated in PE and IUGR but the latter exhibits reduced CT proliferation and this perturbed steady state leads to smaller trophoblast volumes and surfaces; [c] $\mathrm{O}_{2}$ diffusive conductances alter in various pregnancy complications, including IUGR and PE+IUGR; [d] fetal weight is diminished, but diffusive transport increases, in placentas from mice exposed to urban air pollution. Finally, innovations in quantitative immunoelectron microscopy (immunoEM) have produced a portfolio of methods for revealing non-random distributions of marker gold particles in different cellular compartments and for testing whether patterns shift following experimental manipulation. Recently, the methods have been extended to study tissue and cellular distributions of nanoparticles.
\end{abstract}

Keywords: functional morphology, immunoEM, molecule, organ, placenta, stereology.

\section{INTRODUCTION}

A valuable characteristic of stereology is its ability to provide functionally-relevant quantitative information at different levels of structural organization. Coupled with appropriate sampling procedures, stereological estimation permits 3D quantification of the sizes, spatial contents and relationships of molecules, organelles, cells, tissues, organs and organisms (Howard and Reed, 2005). Over the past 20 years, technical developments have improved these procedures and formalised optimal methods. During the past 30 years, with the aid of many excellent colleagues, I have applied established methods to study organ function and developed novel methods for studying subcellular localization of interesting marker probes.
Using the placenta as our organ of interest, we have identified how stereology can be applied to quantify relevant processes (including villous growth and maturation, trophoblast turnover, fetoplacental angiogenesis and passive diffusion) and to test whether or not they are compromised in various complicated pregnancies. Studies on villous volumes, surface areas and lengths in human placentas have shown that growth and maturation involve differential growth of capillaries and that fetoplacental angiogenesis is biphasic with marked changes in vascular content and arrangement occurring around mid-gestation (Mayhew, 2002). Counting nuclei with physical disectors has shown that capillary growth is associated with increased numbers of endothelial cells and that trophoblast is a continuously-renewing epithelium involving CT cell 
mitosis, recruitment into ST and subsequent differentiation and extrusion of syncytial fragments (Mayhew, 2001). Whilst the numerical ratio of ST:CT nuclei remains stable (about 10:1) through most of gestation, trophoblast gradually becomes thinner because its surface area expands faster than its volume. Perhaps surprisingly, the integration of these processes results in changes in total $\mathrm{O}_{2}$ diffusive conductance which match the growing fetal mass (Mayhew, 2006). Similar processes are reproduced during development of the murine definitive placenta (Coan et al., 2004).

Some of these processes are compromised in pregnancy complications, notably $\mathrm{PE}$ and IUGR (Mayhew et al., 2003, 2004, 2007). In particular, we have found that:

a) villous and fetoplacental vascular growth are compromised in pregnancies accompanied by $\mathrm{PE}$ and IUGR. In pure PE, quantities are similar to those seen in normal pregnancies but placental tissue contents are reduced in pure IUGR and in PE+IUGR;

b) alterations in trophoblast turnover differ between PE and IUGR. Whilst trophoblast extrusion rates are higher in PE and IUGR, rates of CT proliferation decrease in IUGR and this leads to reduced volumes and surfaces of trophoblast;

c) $\mathrm{O}_{2}$ diffusive conductances are altered in various pregnancy complications including IUGR. However, specific conductances (related to fetal mass) do not alter significantly in PE or IUGR.

More recently, using experimental mice, we have shown that maternal vascular growth is diminished, but total and specific conductances of the intervascular barrier increase, in placentas from pregnant mice exposed to particulate matter in urban air pollution (Veras et al., 2008). Finally, innovations in quantitative immunoEM have resulted in a set of methods for revealing non-random distributions of marker gold particles in different cellular compartments and testing whether or not distribution patterns shift following experimental manipulation (Mayhew and Lucocq, 2008a; Mayhew, 2009). These methods combine random sampling procedures with stereological event counting to establish the expected patterns of random labelling. Observed and expected distributions are compared by means of relative labelling indices $(\mathrm{RLI}=$ 1 when compartments label randomly) and chi-squared $\left(\chi^{2}\right)$ analyses. These methods have been extended to study intracellular distributions of nanoparticles (Mühlfeld et al., 2007a,b) and membrane surface distributions of virus particles (Yashchenko V, personal communication, 2008). Shifts of labeling between compartments may be undertaken by contingency table analysis using observed counts of gold particles (Mayhew and Lucocq, 2008a).

\section{EXPERIMENTAL FINDINGS}

\section{WHOLE-ORGAN LEVEL}

Placental growth. Findings are illustrated by reference to the human placenta subjected to multistage systematic uniform random sampling (Mayhew, 2008). This transient organ serves the needs of the developing embryo and fetus. As in the pulmonary lung, this occurs by expanding exchange surface areas and minimising diffusion distances. Exchange surfaces expand in two phases by the creation and differentiation of tree-like ramifications (Benirschke and Kaufmann, 2000; Kaufmann et al., 2004). Early in gestation, the main branches (stem and immature intermediate villi) are established and, starting around mid-gestation, a multitude of fine peripheral branches (mature intermediate and terminal villi) rapidly proliferates. At term, the finer branches have an extensive surface $\left(>10 \mathrm{~m}^{2}\right)$ and small calibre $(40-100 \mu \mathrm{m})$ and are crucial to transplacental exchanges. They comprise an outer trophoblastic epithelium which surrounds a mesodermal stroma containing fetoplacental capillaries. The epithelium is composed of proliferative CT cells and a superficial terminally-differentiating ST. In the third trimester, the main layers interposed between maternal and fetoplacental circulations are ST, scattered CT cells, epithelial basal laminae, variable amounts of stroma and vascular endothelium. Local attenuation of trophoblast, especially overlying peripheralised and dilated capillaries, creates vasculosyncytial membranes (Benirschke and Kaufmann, 2000) and reduces effective diffusion distances (harmonic mean about $4 \mu \mathrm{m}$ ). This results in a variably-thick intervascular barrier.

Growth of villi depends on fetoplacental angiogenesis which is also biphasic with an initial phase of capillary branching succeeded by one of greater nonbranching angiogenesis (Benirschke and Kaufmann, 2000; Mayhew, 2002; Kaufmann et al., 2004). The benefit of branching angiogenesis is that it reduces resistance to blood flow. The potential disadvantage of non-branching angiogenesis is partly offset by the fact that fetal capillaries develop local sinusoid-like dilations. Angiogenesis is partly driven by endothelial cell proliferation and intercalation of endothelial progenitor cells (Charnock-Jones et al. 2004). Phasic changes in indices of villous capillarization, notably capillary:villus length ratios (Mayhew, 2002), are consistent with the notion that fetoplacental angiogenesis influences villous differentiation. 
In the definitive mouse placenta, the labyrinth lies close to the chorionic plate and contains irregularlyshaped maternal vascular spaces and a network of fetoplacental capillaries. The two circulations are separated by a superficial layer of CT cells and two layers of ST. Coan et al. (2004) have shown that the placenta reaches its maximum size by E16.5 with the labyrinth continuing to expand thereafter. Volumes and surfaces of the maternal vascular spaces expand rapidly until E16.5 but growth of fetal capillaries continues from E12.5 to E18.5. The changes in fetal capillary volumes arise through increases in total length and reductions in mean cross-sectional area. Trophoblast volume also increases and, coupled with the growth in maternal and fetal exchange surface areas, leads to significant attenuation of the intervascular barrier between E14.5 and E16.5 (Coan et al. 2004).

Growth of peripheral (intermediate and terminal) villi and their fetoplacental microvessels alters in pregnancies complicated by PE and IUGR (Mayhew et al., 2003; 2004; Egbor et al., 2006). In PE not accompanied by IUGR, structural quantities (global volumes, surfaces and lengths) are similar to those seen in normal pregnancies although subject-to-subject variability, expressed as coefficient of variation $(\mathrm{CV})$, tends to be less (Table 1). Tissue contents are reduced in pure IUGR and PE+IUGR and CV values tend to be greater suggesting that there is maladaptation of villi to IUGR.

Placental oxygen transport. As it grows, the fetus requires more $\mathrm{O}_{2}$ from the mother. The ability of the placenta to deliver $\mathrm{O}_{2}$ involves passive diffusion and is determined partly by the physical dimensions (surface areas and effective diffusion distances) of the intervascular barrier and can be expressed as a total conductance (Mayhew et al., 1993; Ansari et al. 2003; Mayhew, 2006). Whilst barrier thickness is inversely proportional to diffusive conductance, it is better to estimate a harmonic rather than an arithmetic mean thickness because the former deals more effectively with the reality of varying local thicknesses.

In human placenta, $\mathrm{O}_{2}$ dissociates from haemoglobin in maternal erythrocytes and crosses maternal blood plasma, trophoblast, fetal endothelium and plasma before binding to haemoglobin in fetal erythrocytes. These six tissue compartments offer resistances to diffusion and can be summed to obtain a total resistance, the reciprocal of which is total conductance (Mayhew et al., 1993). Serial resistances can be calculated from vascular space volumes and $\mathrm{O}_{2}$-haemoglobin chemical reaction rates, surface areas, harmonic mean thicknesses and permeability coefficients. The composition of the intervascular barrier of mouse placenta is different from that of the human placenta and estimates of diffusive conductance have been confined to the barrier itself rather than the placenta as a whole (Coan et al. 2004; Veras et al. 2008). Mass-specific conductances can be calculated by relating total conductances to fetal weight (Mayhew et al., 1993; Coan et al. 2004).

In reality, several factors (e.g. vascular shunts and local perfusion:diffusion ratio inequalities) reduce the effectiveness of the placenta for passive diffusion. Consequently, this approach provides the maximal diffusive conductances achievable under optimal conditions. Nevertheless, estimates have real comparative worth. In human and mouse placentas, $\mathrm{O}_{2}$ diffusive conductances are matched to fetal weight despite placental compartments showing different growth trajectories (Mayhew et al. 1993; Coan et al. 2004; Mayhew, 2006). In fact, the regression line of log intervascular conductance against log fetal weight for human placenta has a slope of 1.04 and this value is not significantly different from 1 (Mayhew, 2006). Studies on mouse placenta (Coan et al. 2004) also suggest that the intervascular conductance is commensurate with changes in fetal weight (slope 0.93). Interestingly, when combined with human findings, the results further suggest that mass-specific conductances are similar across species.

Table 1. Group means (CV\%) for growth measures of villi and fetal capillaries in human placenta. Two-way ANOVA revealed significant effects of IUGR on all variables (based on data in Mayhew et al., 2003, 2004).

\begin{tabular}{lllll}
\hline Variable & Controls $(\mathrm{n}=9)$ & PE $(\mathrm{n}=5)$ & IUGR $(\mathrm{n}=5)$ & PE+IUGR $(\mathrm{n}=5)$ \\
\hline Villous volume, $\mathrm{cm}^{3}$ & $219(26 \%)$ & $179(21 \%)$ & $130(43 \%)$ & $105(43 \%)$ \\
Villous surface area, $\mathrm{m}^{2}$ & $11.1(18 \%)$ & $9.4(4.3 \%)$ & $6.6(50 \%)$ & $5.2(46 \%)$ \\
Villous length, $\mathrm{km}$ & $60.6(33 \%)$ & $45.3(18 \%)$ & $30.9(51 \%)$ & $26.7(41 \%)$ \\
Capillary volume, $\mathrm{cm}^{3}$ & $65.5(40 \%)$ & $46.9(17 \%)$ & $35.3(55 \%)$ & $28.0(55 \%)$ \\
Capillary surface, $\mathrm{m}^{2}$ & $10.8(39 \%)$ & $8.2(22 \%)$ & $5.4(58 \%)$ & $3.8(44 \%)$ \\
Capillary length, $\mathrm{km}$ & $233(42 \%)$ & $172(11 \%)$ & $110(57 \%)$ & $90(55 \%)$ \\
Trophoblast volume, $\mathrm{cm}^{3}$ & $67.9(21 \%)$ & $67.3(6 \%)$ & $50.3(38 \%)$ & $32.5(47 \%)$ \\
\hline
\end{tabular}


Recently, we have shown that total diffusive conductances in cases of IUGR and PE+IUGR are lower than those in uncomplicated or PE pregnancies (Mayhew et al., 2007). We have also found that ultrasound grading of placentas at 31-34 weeks of gestation does not predict organ function at term expressed in terms of diffusive conductance (Yin et al., 2009). In addition, exposing dams to particulate urban air pollution is associated with increases in intervascular diffusive conductances despite which both fetal weight and maternal vascular growth are compromised (Veras et al., 2008).

\section{TISSUE AND CELL LEVELS}

Trophoblast turnover. Disector-based analyses have demonstrated that, in human terminal villi, nuclei of trophoblast, stroma and vascular endothelium continue to increase in number during pregnancy and in a roughly concentric manner (about $40 \%$ trophoblastic, $48 \%$ stromal and $12 \%$ endothelial nuclei). From at least 12 weeks, ST nuclei outnumber CT nuclei by about 10:1 and nett production rates equate to about 14 million (ST) and 1.4 million (CT) per hour (Simpson et al., 1992). CT cells proliferate and some of their daughters subsequently fuse into the overlying ST wherein their nuclei undergo a sequence of differentiation which includes a longer preapoptotic and shorter apoptotic phase. Eventually, apoptotic nuclei are sequestered within nucleus-rich syncytial knots which are extruded into the maternal uteroplacental circulation (Mayhew, 2001). These events demonstrate that trophoblast is a continuouslyrenewing epithelium, the sub-compartments of which exist in a protracted steady state.

Alterations in trophoblast turnover also differ between PE and IUGR (Huppertz et al., 2003; Goswami et al., 2006; Heazell et al., 2006). Rates of trophoblast extrusion are accelerated in PE and IUGR and, in PE, the accelerated turnover is accompanied by a shift from apoptosis towards necrosis. However, in IUGR, CT proliferation and recruitment do not match the rates of loss from ST and this explains the reduced volumes and surfaces of trophoblast seen in IUGR (Table 1; see also Mayhew et al., 2003).

\section{SUBCELLULAR AND MOLECULAR LEVELS}

Immunocytochemical localisation. Developments in quantitative immunoEM have led to a portfolio of methods for revealing non-random distributions of marker gold particles in different cell compartments and testing whether or not distributions alter after experimental manipulation. The methods combine random sampling procedures, stereological event counting and inferential statistics to establish and test for random patterns of labelling.

To study the distribution of gold label between compartments within a cell, observed and predicted distributions are compared by means of relative labelling indices (RLI) and $\chi^{2}$ analyses. Table 2 illustrates the method with a dataset representing the distribution of a gold-labelled protein located in surfaceoccupying compartments (membranes). First, the relative surface areas of different membrane compartments are estimated stereologically by counting intersections with random test lines. Observed gold counts are then used to calculate compartmental labelling densities $\left(\mathrm{LD}_{\mathrm{obs}}\right)$ expressed simply as gold particles per intersection. For the cell as a whole, the membrane labelling density represents the value to be expected $\left(L_{\text {exp }}\right)$ if all membranes were labelled simply by virtue of their relative surface areas. By dividing observed by expected densities, the RLI is obtained and indicates the degree to which a membrane is labelled in comparison to random labelling. Observed and expected gold counts are used to calculate $\chi^{2}$ values for each compartment and for the cell as a whole. In this example, total $\chi^{2}$ is 172.12 and, for 4 degrees of freedom, $\mathrm{P}<0.001$, indicating that observed and expected gold counts are different. Only the secretory granule membranes meet the twin criteria for preferential labeling, i.e., RLI $>1$ and partial $\chi^{2}>$ $10 \%$ of total $\chi^{2}$. Similar principles may be used to compare observed and expected densities (expressed as gold particles per test point) for volume-occupying compartments such as mitochondria, lysosomes or Golgi cisternae.

RLI and LD estimators deal effectively with between-compartment labelling when compartments are either all volume-occupiers or all surfaceoccupiers. However, some molecules translocate from membranes to vesicles (or vice versa) and, in such cases, it is desirable to devise estimators which treat all compartments in the same manner. One way forward (Mayhew and Lucocq, 2008b) is to convert linear membrane traces into profile areas by defining an 'acceptance zone' for each trace with an overall width roughly corresponding to the dispersion zone of gold label on either side of the trace. A useful guide is to adopt a zone with a distance from the membrane trace which is twice the diameter of the gold particles being used to label the membrane of interest. The absolute profile area of the acceptance zone covering both sides of the membrane trace can be found by multiplying overall width by the profile length estimated by intersection counting. If necessary, 
the equivalent number of test points can be estimated for any coherent stereological grid of test lines and points. An additional step is to correct for membrane images lost because they are tilted relative to the section plane or electron axis. This can be achieved by counting only those gold particles which are associated with local vertical windows (those membranes which are clearly visible and vertically sectioned). Correction factors can be determined goniometrically or stereologically by estimating the fraction of poorly-imaged membrane (Mayhew and Lucocq, 2008b).

The RLI and LD methods have been applied to study the distribution of caveolin-1 in human term placenta (Mayhew, 2009) and extended recently to study intracellular distribution of nanoparticles (Mühlfeld et al., 2007a,b) and membrane surface distributions of virus particles (Yashchenko, personal communication, 2008). Similar principles have been used to test for randomness in the deposition of fibrin-type fibrinoid (a maternal blood coagulation product) on different regions of the surface of villous trophoblast (Mayhew et al., 2000).

To test for shifts in the distributions of gold labelling between compartments in different groups of cells, or different antigens in the same cells, observed counts of immunogold particles can be compared by contingency table analysis. Table 3 illustrates the method with a dataset representing the distributions of 3 different proteins across 11 compartments. Total $\chi^{2}$ is 302.25 and, for 20 degrees of freedom, $\mathrm{P}<$ 0.001 , indicating that the 3 distributions differ. In particular, protein 3 exhibits more-than-expected gold particles in endosomal vacuoles and melanosomes. This approach has been used to study the distribution of the glucose transporter, GLUT 1, in trophoblast cells exposed to different glucose concentrations (Mayhew and Desoye, 2004).

Table 2. Distributions of gold particles and test line intersections between different membrane compartments and calculation of labelling densities (LD), relative labelling indices (RLI) and chi-squared values. The secretory granule membranes are preferentially-labelled ( $R L I>1$ and a partial chi-squared $>10 \%$ of total).

\begin{tabular}{|c|c|c|c|c|c|c|}
\hline Compartment & $\begin{array}{l}\text { Observed } \\
\text { gold counts }\end{array}$ & $\begin{array}{l}\text { Observed } \\
\text { intersections }\end{array}$ & $\begin{array}{l}\text { Expected } \\
\text { golds }\end{array}$ & $\begin{array}{l}\text { Labelling } \\
\text { density, } \mathrm{LD}_{\mathrm{obs}}\end{array}$ & $\begin{array}{l}\text { Relative Labeling } \\
\text { Index, } \mathrm{LD}_{\text {obs }} / \mathrm{LD}_{\text {exp }}\end{array}$ & $\begin{array}{l}\text { Chi-squared } \\
(\%)\end{array}$ \\
\hline Plasma membrane & 44 & 117 & 133.13 & 0.38 & 0.33 & $59.65(34.7 \%)$ \\
\hline Golgi membrane & 5 & 16 & 18.21 & 0.31 & 0.28 & $9.57(5.6 \%)$ \\
\hline Rough ER membrane & 116 & 101 & 114.92 & 1.15 & 1.01 & $0.01(0.0 \%)$ \\
\hline $\begin{array}{l}\text { Secretory granule } \\
\text { membrane }\end{array}$ & 205 & 90 & 102.40 & 2.28 & 2.00 & $102.80(59.7 \%)$ \\
\hline Residual membrane & 18 & 17 & 19.34 & 1.06 & 0.93 & $0.09(0.0 \%)$ \\
\hline Column Sums & 388 & 341 & 388 & $\mathrm{LD}_{\exp }=1.14$ & 1.00 & $172.12(100 \%)$ \\
\hline
\end{tabular}

Table 3. Observed (expected) distributions of 3 gold-conjugated proteins across different subcellular compartments of the same cell type and calculation of chi-squared values. Protein 3 differs in its distribution: there are more-than-expected gold particles in endosomal vacuoles and in melanosomes. Tbv indicates tubulovesicular morphology, TGN is the trans-Golgi network and MVB are multivesicular bodies.

\begin{tabular}{llllll}
\hline Compartment & Protein 1 & Protein 2 & Protein 3 & Row Sums & Chi-squared \\
\hline Golgi & $9(10.6)$ & $0(9.5)$ & $22(10.9)$ & 31 & $0.23,9.51,11.26$ \\
Tbv(GolgiTGN) & $23(30.7)$ & $40(27.6)$ & $27(31.7)$ & 90 & $1.93,5.55,0.69$ \\
Endosomal vacuoles & $0(8.9)$ & $0(8.0)$ & $\mathbf{2 6}(\mathbf{9 . 2 )}$ & 26 & $8.87,7.98, \mathbf{3 1 . 0 1}$ \\
Tbv(endosomes) & $104(82.9)$ & $88(74.6)$ & $51(85.5)$ & 243 & $5.38,2.42,13.96$ \\
MVB & $0(5.1)$ & $0(4.6)$ & $15(5.3)$ & 15 & $5.12,4.60,17.89$ \\
Tbv(MVB) & $10(6.5)$ & $9(5.8)$ & $0(6.7)$ & 19 & $1.91,1.72,6.69$ \\
Melanosomes & $0(28.3)$ & $9(25.5)$ & $\mathbf{7 4}(\mathbf{2 9 . 2 )}$ & 83 & $28.31,10.65, \mathbf{6 8 . 6 2}$ \\
Tbv(melanosomes) & $64(43.0)$ & $34(38.7)$ & $28(44.4)$ & 126 & $10.28,0.56,6.03$ \\
Lysosomes & $0(2.0)$ & $0(1.8)$ & $6(2.1)$ & 6 & $2.05,1.84,7.16$ \\
Tbv(lysosomes) & $4(2.4)$ & $3(2.1)$ & $0(2.5)$ & 7 & $1.09,0.34,2.46$ \\
Cytoplasmic vesicles & $35(28.7)$ & $41(25.8)$ & $8(29.6)$ & 84 & $1.41,8.99,15.74$ \\
Column Sums & 249 & 224 & 257 & 730 & 302.25 \\
\hline
\end{tabular}




\section{CONCLUDING REMARKS}

This review has illustrated the value of stereology for interpreting functional morphology at different levels of organisation from the molecular to wholeorgan levels. The examples are personal because they reflect the researches undertaken in my laboratories. However, the practical utility of stereology is limited only by the ability to generate suitably-randomised sectional images and identify ingredients of interest on those sections. Apart from basic scientific researches, the capacity to span levels of biological organisation increases the value of these methods for biomedical translational research. The applicability to different species further extends the possibilities to comparative functional morphology.

The chosen examples show how stereology can advance detailed description and understanding of the processes involved in producing an effective placenta capable of supporting the respiratory and nutritive needs of the developing fetus. At least some of these changes seen could not be resolved to the same degree using alternative approaches such as biochemistry or physiology. They have also demonstrated that key processes (fetoplacental angiogenesis, trophoblast turnover and villous growth) are compromised in IUGR and have a detrimental effect on $\mathrm{O}_{2}$ transport capability. It is these changes in IUGR which emphasise the advisability of monitoring fetal growth in cases of PE.

Finally, we have illustrated some new methods for quantitative immunolocalisation at the EM level. These are based on the sound principles of random sampling and efficient and unbiased estimation that apply to other areas of stereological estimation. However, the efficiency of stereology extends beyond expressions of estimator precision and includes the efficiency which arises from improvements in (or limitations of) biological interpretation gained by quantifying structure in $3 \mathrm{D}$ rather than $2 \mathrm{D}$.

\section{ACKNOWLEDGEMENTS}

TMM is supported currently by The Medical Research Council (MRC) and Biotechnology and Biological Sciences Research Council (BBSRC). Heartfelt thanks are due also to all those colleagues and friends who have helped me to advance my researches over the past 3 decades. A special thanks to Prof Ewald Weibel (Berne) who, for my generation, can be justly regarded as the father of stereology in biology/ biomedicine.

\section{REFERENCES}

Ansari T, Fenlon S, Pasha S, O’Neill B, Gillan JE, Green CJ, et al. (2003). Morphometric assessment of the oxygen diffusive conductance in placentae from pregnancies complicated by intra-uterine growth restriction. Placenta 24:618-26.

Benirschke K, Kaufmann P (2000). Pathology of the human placenta. $4^{\text {th }}$ ed. New York: Springer Verlag.

Charnock-Jones DS, Kaufmann P, Mayhew TM (2004). Aspects of human fetoplacental vasculogenesis and angiogenesis. I. Molecular regulation. Placenta 25: 103-13.

Coan PM, Ferguson-Smith AC, Burton GJ (2004). Developmental dynamics of the definitive mouse placenta assessed by stereology. Biol Reprod 70:1806-13.

Egbor M, Ansari T, Morris N, Green CJ, Sibbons PD (2006). Pre-eclampsia and fetal growth restriction: how morphometrically different is the placenta? Placenta 27:727-34.

Goswami D, Tannetta DS, Magee LA, Fuchisawa A, Redman CWG, Sargent IL, et al. (2006). Excess syncytiotrophoblast microparticle shedding is a feature of early-onset pre-eclampsia, but not normotensive intrauterine growth restriction. Placenta 27:56-61.

Heazell AE, Moll SJ, Jones CJ, Baker PN, Crocker IP (2006). Formation of syncytial knots is increased by hyperoxia, hypoxia and reactive oxygen species. Placenta 28, suppl 1:S33-S40.

Howard CV, Reed MG (2005). Unbiased Stereology. Three-Dimensional Measurement in Microscopy. $2^{\text {nd }}$ edn. Abingdon, Oxon: Garland Science/BIOS Scientific Publishers.

Huppertz B, Kingdom J, Caniggia I, Desoye G, Black S, Korr H, et al. (2003). Hypoxia favours necrotic versus apoptotic shedding of placental syncytiotrophoblast into the maternal circulation. Placenta 24:181-90.

Kaufmann P, Mayhew TM, Charnock-Jones DS (2004). Aspects of human fetoplacental vasculogenesis and angiogenesis. II. Changes during normal pregnancy. Placenta 25:114-26.

Mayhew TM (2001). Villous trophoblast of human placenta: a coherent view of its turnover, repair and contributions to villous development and maturation. Histol Histopathol 16:1213-24.

Mayhew TM (2002). Fetoplacental angiogenesis during gestation is biphasic, longitudinal and occurs by proliferation and remodelling of vascular endothelial cells. Placenta 23:742-50.

Mayhew TM (2006). Allometric studies on growth and development of the human placenta: growth of tissue compartments and diffusive conductances in relation to placental volume and fetal mass. J Anat 208:785-94.

Mayhew TM (2008). Taking tissue samples from the placenta: an illustration of principles and strategies. Placenta 29:1-14. 
Mayhew TM (2009). Quantifying immunogold localization patterns on electron microscopic thin sections of placenta: recent developments. Placenta 30:565-70

Mayhew TM, Bowles C, Orme G (2000). A stereological method for testing whether or not there is random deposition of perivillous fibrin-type fibrinoid at the villous surface: description and pilot applications to term placentas. Placenta 21:684-92.

Mayhew TM, Desoye G (2004). A simple method for comparing immunogold distributions in two or more experimental groups illustrated using GLUT1 labelling of isolated trophoblast cells. Placenta 25:580-4.

Mayhew TM, Jackson MR, Boyd PA (1993). Changes in oxygen diffusive conductances of human placentae during gestation (10-41 weeks) are commensurate with the gain in fetal weight. Placenta 14:51-61.

Mayhew TM, Lucocq JM (2008a). Developments in cell biology for quantitative immunoelectron microscopy based on thin sections - a review. Histochem Cell Biol 130:299-313.

Mayhew TM, Lucocq JM (2008b). Quantifying immunogold labelling patterns of cellular compartments when they comprise mixtures of membranes (surface-occupying) and organelles (volume-occupying). Histochem Cell Biol 129: 367-78.

Mayhew TM, Manwani R, Ohadike C, Wijesekara J, Baker PN (2007) The placenta in pre-eclampsia and intrauterine growth restriction: studies on exchange surface areas, diffusion distances and villous membrane diffusive conductances. Placenta 28:233-8.

Mayhew TM, Ohadike C, Baker PN, Crocker IP, Mitchell C, Ong SS (2003). Stereological investigation of placental morphology in pregnancies complicated by pre-eclampsia with and without intrauterine growth restriction. Placenta 24:219-26.

Mayhew TM, Wijesekara J, Baker PN, Ong SS (2004). Morphometric evidence that villous development and fetoplacental angiogenesis are compromised by intrauterine growth restriction but not by pre-eclampsia. Placenta 25:829-33.

Mühlfeld C, Geiser M, Kapp N, Gehr P, Rothen-Rutishauser B (2007a). Re-evaluation of pulmonary titanium dioxide nanoparticle distribution using the "relative deposition index": evidence for clearance through microvasculature. Particle Fibre Toxicol 4:7:1-8.

Mühlfeld C, Mayhew TM, Gehr P, Rothen-Rutishauser B (2007b). A novel quantitative method for analyzing the distributions of nanoparticles between different tissue and intracellular compartments. J Aerosol Med 20: 395-407.

Simpson RA, Mayhew TM, Barnes PR (1992). From 13 weeks to term, the trophoblast of human placenta grows by the continuous recruitment of new proliferative units: a study of nuclear number using the disector. Placenta 13:501-12.

Veras MM, Damaceno-Rodrigues NR, Guimarães Silva RM, Caldini EG, Ribeiro AACM, Mayhew TM, et al. (2008). Particulate urban air pollution affects the functional morphology of mouse placenta. Biol Reprod 79:578-84.

Yin TT, Loughna P, Ong SS, Padfield J, Mayhew TM (2009). No correlation between ultrasound placental grading at 31-34 weeks of gestation and a surrogate estimate of organ function at term obtained by stereological analysis. Placenta 30:726-30 